\title{
Primary Prevention of Allergic Diseases: Current Concepts and Mechanisms
}

\author{
Kerstin Gerhold, MD, Yasemin Darcan, MD, and Eckard Hamelmann, MD
}

\begin{abstract}
Atopic diseases, the new "epidemic of the twenty-first century" and a central health problem of industrial nations, call for the development of innovative primary prevention strategies. The present review provides an overview of current experimental and immunomodulatory procedures and their underlying mechanisms.
\end{abstract}

Key words: asthma, Th1/Th2-cytokines, immunomodulation, mouse model

A s a new "epidemic of the 21st century" causing growing health problems, particularly in industrialized countries, atopic diseases such as hay fever, bronchial asthma, and atopic dermatitis call for the development of innovative primary prevention concepts (Figure 1, Table 1).

Pathophysiology of allergic diseases is based on extreme T helper (Th)2 immune responses to commonly harmless environmental antigens. The key cytokines interleukin (IL)-4 and IL-13 induce immunoglobulin (Ig) class switch in B cells, leading to excessive IgE production with subsequent mast cell activation and mediator release, and IL-5 contributes to development of eosinophilic inflammation and enhances mucus production of the airway epithelia (recently reviewed by Coffmann ${ }^{2}$ ). The reasons for dysregulation and the resulting imbalance in cellular immune responses on allergens are still not certainly identified. Genetic predisposition, especially gene-gene interactions, ${ }^{3}$ seems to be a fundamental factor but does not explain the extensive increase in the incidence and prevalence of atopic diseases within the last 40 years. Numerous environmental triggers might account for this increase, such as altered climate conditions with increasing global warming, resulting in lengthened pollen seasons and thus increased exposure to environmental allergens, or lifestyle factors, such as improved hygiene. ${ }^{4}$ Simple

Kerstin Gerhold, Yasemin Darcan, and Eckard Hamelmann: Department of Pediatric Pneumology and Immunology, Charite, Universitätsmedizinm, Berlin, Germany.

Correspondence to: Clinic of Pediatric Pneumology and Immunology, University Hospital Charite, Campus Virchow Clinic, Augustenburger Platz 1, 13353 Berlin.

DOI $10.2310 / 7480.2007 .00007$ allergen avoidance for primary prevention of allergy appeared not to be practical or sufficient, ${ }^{5}$ and present antiphlogistic therapies with antihistamines or steroids just diminish symptoms for a short time but potentially cause side effects and are not curative. ${ }^{6}$

New immunomodulatory strategies aim to support naturally occurring regulatory mechanisms that may protect against predominant Th2 immune responses and maintain the immunologic balance, thus preventing the development of allergen sensitization as the first step of the atopic march in high-risk children. ${ }^{7}$ Most of these new methods are currently under experimental investigation, and only a few have already been employed in humans. The present review provides an overview of these various immunomodulatory strategies and their principal mechanisms.

\section{Th1/Th2 Concept: Center of Immunomodulatory Prevention Strategies}

Polarization of the adaptive cellular immune response is based on antigen presentation by dendritic cells (DCs) or other antigen-presenting cells (APCs) that leads to differentiation of naive $\mathrm{CD} 4^{+} \mathrm{T}$ cells into $\mathrm{Th} 1$ or $\mathrm{Th} 2$ effector cells. Immature skin or mucosa-associated DCs phagocytize a foreign antigen on its entry site and migrate via blood and lymph to secondary lymphatic organs while they are differentiating to mature APCs. In secondary lymphatic organs, DCs create an immunologic synapse with naive $\mathrm{CD}^{+} \mathrm{T}$ cells: they present the phagocytized and processed antigen in a complex with major histocompatibility complex molecules to the respective T-cell receptor, secrete cytokines, and express costimulatory molecules that interact with specific coreceptors on the $\mathrm{T}$ cell. 


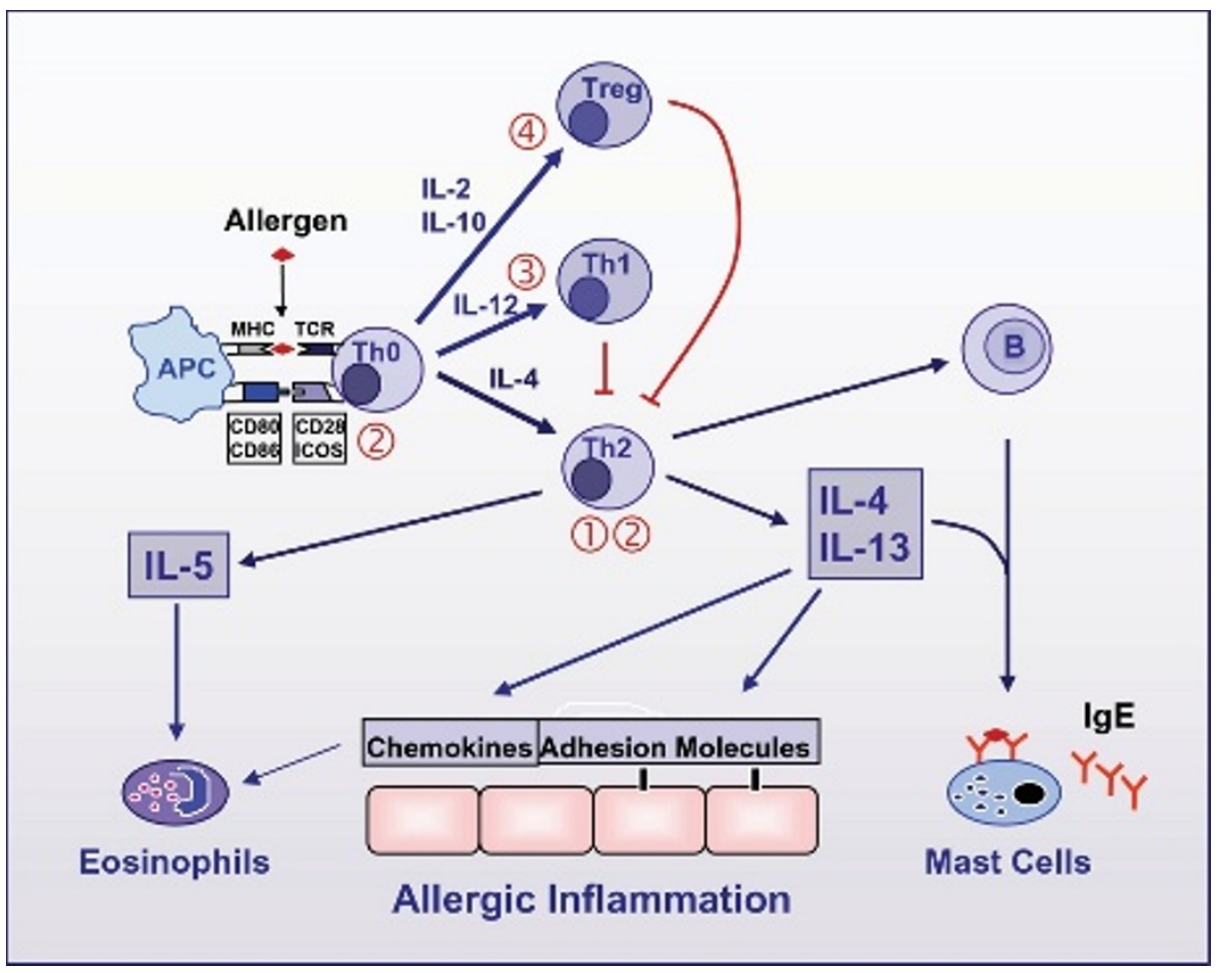

Figure 1. Allergen-induced immune response and new concepts for primary prevention. Allergen-mediated activation of antigen-presenting cells (APCs) induces allergen-specific $\mathrm{T}$ helper (Th) 2 cells. Th2 cells produce Th2 cytokines, resulting in increased production of immunoglobulin $\mathrm{E}$ by $\mathrm{B}$ cells, activation of chemokines and adhesion molecules, and, finally, allergic inflammation. In round brackets, targets for primary prevention concepts, as shown in Table 1.

In the presence of regulatory factors such as thymic stromal lymphopoietin (TSLP), ${ }^{8}$ which is produced by epithelial cells, of the costimulatory proinflammatory molecule OX40 ligand, ${ }^{9}$ and of IL-4, allergen-induced activation of mature $\mathrm{CD} 8 \mathrm{a}^{-}$myeloid DCs of the lungs initiates differentiation of naive $\mathrm{CD} 4{ }^{+} \mathrm{T}$ cells to Th2 cells. IL4 activates cytoplasmic janus kinases (JAKs) 1, 2, and 3 through its two T-cell receptor subsets that phosphorylate tyrosine rests and subsequently activate transcription factor signal transducer and activator of transcription (STAT)6. STAT6 mediates induction of transcription factor GATA-3. Both of them initiate transcription of the Th2 cytokines IL-4, IL-5, and IL-13, most likely through activation of the respective promoter genes. ${ }^{10,11}$

Intracellular pathogens promote mature $\mathrm{CD} 8 \mathrm{a}^{+}$plasmocytoid DCs to produce IL-12, IL-23, and interferon (IFN) $-\gamma$. Binding of IL-12 to the $\beta_{2}$-subset of the IL-12R on $\mathrm{CD}^{+} \mathrm{T}$ cells activates JAK2 and subsequently STAT4. STAT4 activates the IFN- $\gamma$ promoter gene, which probably directly induces production of IFN- $\gamma$. Further, IL-12 is able to intensify Th1 immune responses through activation of mitogen-activated protein kinase (MAPK) p38, resulting again in STAT4 activation. IFN- $\gamma$, which is secreted by mature plasmocytoid DCs and by $\mathrm{T}$ cells in an autocrine pathway, activates the transcription factors STAT1 and subsequently $\mathrm{T}$ box expressed in T cells ( $\mathrm{T}$ bet). As a so-called "master controller," T-bet promotes the Th1 immune response indirectly via suppression of GATA-3. ${ }^{12}$

In terms of the dichotomy of the adaptive cellular immune response first described by Mosmann and colleagues, ${ }^{13}$ the Th1 immune response acts as a natural antagonist of the Th2 immune response. Thus, various prevention concepts aim at generation of Th1 effector cells to suppress Th2 immune responses. At the same time, predominance of Th1 immune responses is believed to trigger development of autoimmune diseases such as type 1 diabetes, autoimmune thyroiditis, or rheumatic diseases. But as recently shown, the rise of autoimmune inflammation depends on IL-17producing Th17 cells. In contrast to former assumptions, Th17 cells do not develop from precursor Th1 cells but represent a third Th cell population, which is directly induced by DCs producing IL-23 and inhibited by both cytokines, IL-4 and IFN- $\gamma$. Therefore, IL-4 and IFN- $\gamma$ prevent development of autoimmune diseases, which has also been increasing within the last 40 years. ${ }^{14,15}$ Use of Th1 cytokines (IFN- $\gamma$, IL-12) in clinical surveys was ineffective or showed high rates of side effects. ${ }^{16}$

\section{Modulation of the Signal Transduction Cascade by Inhibition of Transcription Factors}

Specific blockade of Th2 effector cytokines by monoclonal antibodies is used to treat already existing allergic diseases. 
Table 1. Immunomodulatory Concepts for Prevention of Allergen-Mediated T Helper 2 Immune Response

\begin{tabular}{|c|c|c|c|c|}
\hline No. in Figure 1 & Principle & Target & Mechanism & Examples \\
\hline \multirow[t]{2}{*}{1} & \multirow[t]{4}{*}{$\begin{array}{l}\text { Inhibition of Th2 } \\
\text { cytokine synthesis }\end{array}$} & \multirow[t]{2}{*}{$\begin{array}{l}\text { Transcription factors } \\
\text { of Th2 cytokines }\end{array}$} & $\begin{array}{l}\text { Inhibition of synthesis } \\
\text { of transcription factors } \\
\text { on the level of } \\
\text { transcription }\end{array}$ & $\begin{array}{l}\text { Imiquimod/resiquimod } \\
\text { ODN decoys }\end{array}$ \\
\hline & & & $\begin{array}{l}\text { Inhibition of synthesis } \\
\text { of transcription factors } \\
\text { on the level of } \\
\text { translation }\end{array}$ & $\begin{array}{l}\text { Antisense ODN } \\
\text { siRNA }\end{array}$ \\
\hline \multirow[t]{2}{*}{2} & & \multirow[t]{2}{*}{$\begin{array}{l}\text { Protein kinases of signal } \\
\text { transduction cascade }\end{array}$} & $\begin{array}{l}\text { Inhibition of signal } \\
\text { transduction cascade } \\
\text { following activation } \\
\text { of TCR and/or } \\
\text { costimulatory receptor } \\
\text { molecules }\end{array}$ & Inhibitors of ERK, MEK $1 / 2$ \\
\hline & & & $\begin{array}{l}\text { Inhibition of signal } \\
\text { transduction cascade } \\
\text { on the way to synthesis } \\
\text { of costimulatory } \\
\text { receptor molecules }\end{array}$ & $\begin{array}{l}\text { Inhibitors of ICOS-inducing } \\
\text { protein kinases }\end{array}$ \\
\hline \multirow[t]{4}{*}{3} & \multirow[t]{4}{*}{$\begin{array}{l}\text { Induction of Th1 } \\
\text { immune response }\end{array}$} & \multirow[t]{4}{*}{$\begin{array}{l}\text { Pattern recognition } \\
\text { receptors on APCs }\end{array}$} & Activation of TLR-2 & Mycobacterial antigens \\
\hline & & & Activation of TLR-9 & CpG motifs \\
\hline & & & Activation of TLR-4 & Lipopolysaccharides \\
\hline & & & $?$ & Probiotics \\
\hline 4 & $\begin{array}{l}\text { Induction of tolerance- } \\
\text { inducing Tregs }\end{array}$ & $\begin{array}{l}\text { Transcription factors } \\
\text { of regulatory cytokines? }\end{array}$ & $\begin{array}{c}\text { Induction of Foxp3, } \\
\text { TGF- } \beta \text { and IL-10 }\end{array}$ & $\begin{array}{l}\text { SIT } \\
\text { Parasites }\end{array}$ \\
\hline
\end{tabular}

$\mathrm{APC}=$ antigen-presenting cell; $\mathrm{CpG}=$ cytosine guanine dinucleotide; $\mathrm{ERK}=$ extracellular signal-regulated protein kinase; Foxp3 = forkhead box protein 3; ICOS = inducible costimulator; IL = interleukin; ODN = oligonucleotide; siRNA = small interfering ribonucleic acid; SIT $=$ allergen-specific immune therapy; $\mathrm{TCR}=\mathrm{T}$-cell receptor; $\mathrm{TGF}=$ transforming growth factor; $\mathrm{Th}=\mathrm{T}$ helper; $\mathrm{TLR}=$ Toll-like receptor; $\mathrm{Treg}=$ regulatory $\mathrm{T}$ cell.

On the contrary, molecular concepts aim at inhibition of the distinct transcription factors STAT6 and GATA-3 for primary prevention of allergen-induced sensitization and Th2 immune responses. Antiviral activity of imidazoquinolines such as imiquimod is based on inducing Th1 immune responses in macrophages and DCs that was exploited to antagonize Th2 immune responses. In our mouse model of allergen-induced airway inflammation, local application of the imiquimod derivative resiquimod via the airways after allergen sensitization but prior to airway allergen challenges inhibited development of eosinophilic airway inflammation and airway hyperreactivity that was associated with a shift from a predominant Th2 immune response toward a predominant Th1 immune response. ${ }^{17}$ Induction of T-bet and suppression of GATA-3 were recently described to be the fundamental and protective mechanisms of imidazoquinolines. ${ }^{18}$ Inhibition of Th2-inducing transcription factors can also be performed by so-called "gene silencing," the inhibition of distinct gene transcription. Oligonucleotide (ODN) decoys competitively inhibit binding of transcription factors at the deoxyribonucleic acid (DNA) of specific promoter genes and therefore inhibit transcription of respective genes. Indeed, inhibition of STAT6 by means of ODN decoys did diminish proliferation of murine and human Th2 cells in vitro ${ }^{19}$ and did suppress IgE synthesis and development of the late-phase inflammatory response in vivo in a mouse model of atopic dermatitis. ${ }^{20}$

Although STAT1 directs Th1 immune responses, it also supports development of allergen-induced airway inflammation by enhancing expression of the costimulatory molecule CD40 on APCs and B cells. CD40 interacts with 
CD40L on $\mathrm{T}$ cells and activates them to produce Th2 cytokines. In accordance, intranasal application of STAT1inhibiting ODN decoys did diminish Th2 cytokine production and expression of IL-4-dependent vascular cell adhesion molecule (VCAM)-1 on endothelial cells, which is known to promote leukocyte infiltration of the airways and therefore did prevent development of allergeninduced airway disease in sensitized mice. ${ }^{21}$ Further experimental studies are required to analyze the effects of STAT1 on allergen sensitization.

Competitive inhibition of production of transcription factors and cytokines at the ribonucleic acid (RNA) level might also result in diminished Th2 cytokine production (recently reviewed by Popescu ${ }^{22}$ ). Specific antisense ODNs containing 15 to 20 ODNs activate ribonuclease $\mathrm{H}$, which splits the RNA rest out of DNA-RNA double strands and therefore degrades target messenger RNA, or antisense ODNs inhibit translation via steric blockade of ribosomes. In fact, in a mouse model, local application of specific antisense ODNs did diminish expression of GATA-3, which resulted in dramatically suppressed Th2 cytokine production and allergen-mediated airway inflammation. ${ }^{23}$ In contrast, suppression of STAT6 by antisense ODN decoys showed divergent therapeutic effects in vitro and in vivo. ${ }^{24,25}$

Compared to antisense ODN decoys, the small interfering ribonucleic acid (siRNA) technique promises to be more efficient. Specific endonucleases, so-called "dicer enzymes," split long double-strand RNA into siRNA containing 21 to 23 nucleotides. Alternatively, synthesized siRNAs are commercially available. siRNAs are integrated into the RNA-induced silencing complex (RISC), which contains helicases, endonucleases, and exonucleases. RISC degradates specifically target RNA molecules by means of the antisense strand of siRNA to interrupt protein biosynthesis. ${ }^{26}$ Trian and colleagues recently showed that siRNA inhibited expression of mast cell protease-activated receptor (PAR)-2 in human airway smooth muscle cells in vitro. ${ }^{27}$ PAR-2 is probably involved in activating airway smooth muscle cells; therefore, it might provoke airway obstruction and hyperreagibility in bronchial asthma. ${ }^{27}$ At present, we are analyzing in our mouse model of allergen-induced airway inflammation whether local application of siRNA suppresses expression of STAT6 and GATA-3 and subsequently inhibits allergen-induced airway inflammation.

\section{Modulation of the Signal Transduction Cascade by Inhibition of Protein Kinases}

Receptor-dependent cytoplasmatic protein kinases are responsible for phosphorylation and activation of tran- scription factors; thus, they fundamentally control differentiation of naive $\mathrm{CD}^{+}{ }^{+} \mathrm{T}$ cells in Th1/Th2 effector cells and synthesis of mediators, inducing development of allergen-induced inflammation. Inhibition of JAKs, which take part in differentiation of both Th1 and Th2 effector cells, might result in unspecific effects. In contrast, the extracellular signal-regulated protein kinase (ERK), which belongs to the MAPK, mediates activation of the eosinophilic IL-5R and eotaxin-R, initiating accumulation and degranulation of eosinophils in the airways. ${ }^{28,29}$ Systemic application of a specific inhibitor (UO126) inhibited ERK through competitive inhibition of upstream MAPK/ERK-kinase (MEK)1/2 and suppressed allergeninduced IgE production, VCAM-1 expression in lungs, mucus production in the airway, and airway hyperreactivity in mice. ${ }^{30}$

Th2-cell differentiation requires further costimulatory signals, particularly interactions between CD28 and inducible costimulator (ICOS) on T cells on the one hand and their ligands CD80/86 and ICOS-L on DCs, B cells, and other APCs on the other hand. ${ }^{31}$ ICOS acts through activation of MAPK, ERK, and Jun NH2-terminal kinase (JNK). Systemic application of U0126 or SP600125 selectively inhibited ERK or JNK, which, respectively, prevented local allergen-mediated Th2 immune responses and eosinophilic airway inflammation in allergen-sensitized mice following airway allergen challenges. ${ }^{32}$ ICOS transcription is regulated by two independent pathways, the Fyncalcineurin-NFATc2 pathway and the MEK2-ERK1/2 pathway. ${ }^{33}$ Thus, expression of the proinflammatory costimulatory molecule ICOS might be diminished by inhibiting members of these pathways, such as the protein kinase Fyn, the transcription factor nuclear factor of activated $\mathrm{T}$ cell (NFAT)c2 or MEK2/ERK1/2. Methods might include direct kinase inhibitors or "gene silencing" techniques.

\section{Modulation of Immune Responses through Stimulation of Innate Immunity}

DC activation by foreign antigens represents the first step on the way toward T-cell activation and maturation and therefore the first step on the way toward allergen sensitization. Most allergens are immunologic inert proteins that typically do not induce inflammatory responses but allergen-specific tolerance. However, the presence of so-called "danger signals" such as proteolytic enzyme activity of allergens themselves or microbial antigens leads to DC activation. Particularly, DCs express pattern recognition receptors (PPRs) such as Toll-like receptors (TLRs) for microorganism-associated molecular 
patterns (MAMPs) that are invariant and consistent molecular structures of bacteria and other microorganisms. PPR activation induces MAMP-dependent signal transduction and activation of transcription factor nuclear factor (NF)- $\kappa \mathrm{B}$ and of MAPK, followed by transcription of proinflammatory cytokines such as tumour necrosis factor (TNF)- $\alpha$, IL-6, und IL-12 and expression of costimulatory molecules such as CD40 and CD80/CD86 (recently reviewed by Kaisho and Akira ${ }^{34}$ ). Regular development of the immune system and the balance of adaptive Th1/ Th2 immune responses is probably based mainly on natural exposition to microbial antigens as TLR ligands via the gastrointestinal tract, skin, and airways or on several infectious diseases during early infancy and childhood. A variety of immunomodulatory prevention concepts attempt to reconstitute the natural balance of the adaptive immune response by specific activation of PPRs by means of microbial antigens.

\section{Mycobacterial Antigens}

Mycobacterial antigens such as lipoproteins activate TLR-2 in complex with TLR-1 and TLR-6 or TLR-4; induce production of IL-12, TNF- $\alpha$, IL-10, and IL-15; and initiate development of Th1 effector cells. ${ }^{35}$ In numerous mouse models, vaccination with live or inactivated pathogenic or apathogenic Mycobacteria prevented development of allergen-mediated sensitization and airway inflammation. ${ }^{36-39}$ Recent clinical trials showed a therapeutic effect such as subcutaneous injection of heat-inactivated Mycobacteria bovis bacille Calmette Guérin on pre-existing asthma in adults $^{40}$ or intradermal application of Mycobacterium vaccae on moderate or severe atopic eczema in children. ${ }^{41}$ Nevertheless, primary preventive effects of Mycobacteria on atopic diseases in humans need to be further investigated.

\section{CpG motifs}

Unmethylated cytosine guanine dinucleotides (CpGs) are common components of prokaryotic bacterial or viral DNA; they are also synthetically produced (CpG motifs). CpGs are incorporated by DCs via endocytosis; they bind and activate cytosolic TLR-9 and induce activation of NF$\kappa \mathrm{B}$, followed by secretion of type I interferons, IL-12, IFNinducing protein 10, and other cytokines and chemokines. The resulting innate Th1 immune response is short and limited to proliferating $\mathrm{T}$ cells; it is not able to modulate memory Th2 cells. ${ }^{34}$ Further, CpG motifs activate the tryptophan-degrading enzyme indolamine-2,3-deoxygenase (IDO) via the STAT1 pathway in $\mathrm{CD}^{+} 9^{+} \mathrm{DCs}$.
Intracellular lack of tryptophan and its metabolites causes toxic and other unknown effects, causing diminished $\mathrm{T}$ cell proliferation and immune suppression. Thus, CpG motifs support development of regulatory $\mathrm{T}$ cells (Tregs). ${ }^{42}$ Accordingly, they induced Th1 cells and/or Tregs that inhibited Th2 immune responses and prevented allergen-induced sensitization and airway inflammation in many animal models and clinical trials (lately reviewed by Racila and Kline ${ }^{43}$ ). At present, CpG motifs are more and more used as adjuvants for allergen-specific immune therapy (SIT), even in humans. CpG motifs are conjugated with allergens; local or systemic administration of these conjugates generates allergen-specific long-lasting adaptive Th1 immune responses, induces Tregs, and probably also stimulates memory Th2 cells to shift into Th1 effector cells after further allergen contacts. ${ }^{44}$

\section{Lipopolysaccharides}

The so-called "farming effect" belongs to the bestdescribed environmental factors that are associated with a diminished risk of atopic diseases. ${ }^{45}$ It is based on intensive exposure to organic dust and thus to a variety of microbial antigens in stables on farms from early infancy on. Peters and colleagues recently confirmed protective properties of organic dust from stables with regard to allergen-mediated sensitization and airway inflammation in a mouse model. ${ }^{46}$ Several experimental studies in mice and humans have analyzed, in particular, the immunomodulatory allergy-preventing effects of lipopolysaccharides (LPSs), the cell wall component of gram-negative bacteria and an important ingredient of organic dust. In serum, LPSs bind their soluble receptors lipopolysaccharide-binding protein (LBP) and CD14 and activate TLR-4; LBP and CD14 catalyze TLR-4 activation.

TLR-4 activation activates through the intracellular adaptor molecule MyD88-associated cytoplasmatic protein kinases such as IL-1 receptor-associated kinase (IRAK) 4 and others (TRAF6, TAK1, IKK $\beta$ ), which leads to I $\mathrm{B}$ phosphorylation and finally to $\mathrm{NF}-\kappa \mathrm{B}$ activation. ${ }^{34}$ Epidemiologic studies suggested that polymorphisms for CD14 and TLR-4 resulting in reduced responsiveness of DCs on LPSs are associated with an increased risk of developing atopic diseases. ${ }^{47}$ In our own work in adult mice, local and systemic application of LPSs later suppressed allergen-mediated sensitization and airway inflammation in an IL-12-dependent way. ${ }^{48}$ In neonatal mice, repetitive exposure to simple aerosolized LPSs did not prevent subsequent allergen sensitization, but in combination with allergen-induced mucosal tolerance, 
LPSs elicited an unspecific Th1 immune response, which might diminish the susceptibility of organisms to a variety of environmental allergens. ${ }^{49}$

Further, Wang and McCusker showed in a similar model that repetitive exposure of neonatal mice to LPS and ovalbumin led to development of tolerance-inducing Tregs in later sensitized mice. ${ }^{50}$ Prenatal initiated and postnatal continued exposition to aerosolized LPS inhibited development of allergen-induced sensitization and airway inflammation in the offspring that was associated with a shift from a predominant Th2 immune response toward a predominant Th1 immune response and was most likely mediated by upregulation of the LPS receptors LBP, CD14, TLR-2, and TLR-4, as well as of the Th1 regulatory transcription factor T-bet. ${ }^{51}$

At present, we are investigating in a prospective, double-blind, placebo-controlled, interventional trial in high-risk infants the potentially preventive effect of orally given apathogenic Escherichia coli strains on the development of atopic dermatitis within the first 7 months of life.

\section{Probiotics}

Colonization of the gut by commensal microbes within the first months of life represents the first and probably most important stimulus for the development of the gutassociated immune system, the largest organ-associated immune system. Composition of the gut flora might influence allergen sensitization decisively since epidemiologic observations demonstrated that countries with a high or low prevalence of allergic diseases and atopic and nonatopic individuals showed different microbial strains in the gut, ${ }^{52}$ and oligosaccharides (prebiotics) might prevent allergies by supporting the growth of distinct microbes. ${ }^{53}$ Thus, at present, animal models and clinical trials are used to elucidate the potentially preventive effects of probiotics, living apathogenic bacteria with health-supporting effects. Indeed, in a prospective clinical trial, Lactobacillus rhamnosus, which was given orally during pregnancy and further on during the first months of life, inhibited manifestation of atopic dermatitis in high-risk infants. ${ }^{54}$ The probiotics employed are lactobacilli and bifidobacteria in particular, which are acid resistant and adherent to gut mucosa and further colonize the gut. The mechanisms are unclear. In neonatal mice, probiotics induced development of transforming growth factor (TGF)- $\beta$ producing $\mathrm{T}$ cells, resulting in diminished IgE and Th2 cytokine production $^{55}$; another clinical trial showed enhanced Th2antagonizing IFN- $\gamma$ production. ${ }^{56}$ Increased permeability of gut epithelia for allergens, which was shown for children with atopic dermatitis, is also suggested to cause allergen sensitization.

Distinct gut bacteria produce toxic metabolites such as D-lactic acid or acetaldehyde, which inhibit adenosine triphosphate-dependent synthesis of the epithelial cytoskeleton, resulting in defective barrier functions. In young infants, these metabolites accumulate even more as a consequence of immature degrading enzymes. Probiotics, which do not induce toxic metabolites, might provide a balance of the gut flora and compensate for toxic effects, such as breast milk. ${ }^{57}$

\section{Modulation of Immune Responses by Tolerance Induction}

The immune system physiologically does not respond to self-molecules or harmless environmental antigens. Tregs are thought to mediate this phenomenon of antigen-specific tolerance. Natural Tregs develop in the thymus, express constitutively CD25 (IL-2R $\alpha$ chain) and the transcription factor forkhead box protein 3 (Foxp3), and act in an antigenindependent manner immunosuppressively. In the periphery, a xenogeneic group of adaptive antigen-specific Tregs (aTregs) develop from still unknown $\left(\mathrm{CD} 25^{-}\right)$precursor cells in response to foreign antigens. ATregs become CD25 $5^{+}$ during their development; only some of them express Foxp3, especially following activation through $\mathrm{CD} 3, \mathrm{CD} 28$, and TGF- $\beta .^{58}$ IL-2 is a decisive growth factor for Tregs; CD28 acts as a costimulatory factor ${ }^{58}$; Foxp 3 forms a complex with histone acetyltransferases, histone deacetylases, and chromatin remodeling factors, and inhibits acetylation of histones that results in stopping of DNA transcription as the first step in T-cell proliferation and differentiation. ${ }^{58}$ Akdis and colleagues first described diminished numbers of Tregs in atopic patients. ${ }^{59}$ Thus, an imbalance between Th2 (and Th1) cells on the one hand and Tregs on the other hand might be responsible for the development of atopic diseases, and immunomodulatory prevention concepts focus on induction of Tregs. The Foxp3 complex itself might be a target; inhibitory factors of histone deacetylases mediate stopping of the cell cycle, diminish cytokine expression, and increase apoptosis, but low target specificity causes serious side effects. At present, more specific Foxp3-associated molecular targets are being extensively investigated to modulate the effects of the Foxp3 complex. ${ }^{58}$

Myeloid and plasmocytoid, immature and mature DCs induce aTregs by producing anti-inflammatory cytokines, particularly IL-10. In a positive feedback mechanism, IL-10 from DCs and IL- 10 and TGF- $\beta$ produced by Tregs initiate the development of tolerogenic DCs. ${ }^{60}$ Further, Tregs 
suppress expression of costimulatory molecules such as CD80/CD86 on maturing DCs. Thus, antigen-activated Tregs are able to inhibit sufficient presentation of further antigens by the same DC. ${ }^{61}$ Allergens in higher doses than required only for allergen sensitization activate $\mathrm{CD} 8^{-}$ myeloid DCs that initiate differentiation of aTregs through their costimulatory molecule ICOS-L and transient production of IL-10.

At present, allergen-specific immunotherapy (SIT) represents the only established curative but merely secondary preventive and antigen-specific therapy for allergic diseases. Subcutaneous applications of increasing doses of allergen over 3 to 5 years induce allergen-specific Foxp $3^{+}$Tregs, which express surface molecules such as cytotoxic $\mathrm{T}$ lymphocyte antigen (CTLA)-4 und programmed death (PD)- 1 and secret IL-10 and TGF- $\beta$. Therefore, these cells induce a lifelong allergen-specific tolerance through intensive immunosuppressive and anti-inflammatory properties. ${ }^{62}$ CTLA-4 of these Tregs also activates mature DCs via CD80/CD86, which consequently express IDO and may suppress T-cell functions the other way round. ${ }^{61}$

Following mucosal allergen exposition via the airways, plasmacytoid DCs are activated, which generate Tregs and cause allergen-specific mucosal tolerance in mice. ${ }^{49,62}$ Our own preliminary data showed that repetitive exposures of pregnant mice to aerosolized allergen consistently prevented later allergen sensitization and airway inflammation in the offspring associated with diminished allergenspecific T-cell responses in vitro and development of IFN- $\gamma$-producing $\mathrm{T}$ cells (unpublished data).

Heat-inactivated Listeria monocytogenes, which was given as an adjuvant together with an allergen, activated mature $\mathrm{CD} 8^{+}$plasmacytoid DCs to produce IL-10 and IL12 , resulting in development of IL-10- and IFN- $\gamma$ producing allergen-specific Tregs. These Th1-like Tregs expressed Foxp3 and later prevented allergen-mediated airway hyperreactivity in mice. ${ }^{63}$

\section{Modulation of Immune Responses by Parasites}

During their acute infectious state, helminthes secrete proteases that act as virulent factors and induce a strong Th2 immune response and a massive unspecific IgE production in the host. Further, proteases act as "danger signals" and activate DCs that might promote allergen sensitization. ${ }^{64}$ Additionally, parasite antigens such as tropomyosins might show cross-reactivity with allergens, resulting in enhanced allergen sensitization. ${ }^{65}$ In contrast, the anti-inflammatory effects of helminthes in the chronic state might be responsible for inverse correlations between parasitic and allergic diseases. ${ }^{66}$ The anti-inflammatory property of helminthes is more and more used for immunomodulatory therapeutic and prevention concepts, although the underlying mechanisms have not been clarified. Both DCs and APCs, as well as $\mathrm{CD}^{+} \mathrm{T}$ cells, might play a key role. According to experimental data, helminthes induce Foxp $3^{+}$IL-10- and TGF- $\beta$-producing Tregs that inhibit development of allergen-mediated sensitization and airway inflammation in mice. ${ }^{67,68}$ Helminthes also induce $\mathrm{CD}^{+}$natural killer $\mathrm{T}$ cells, a subgroup of $\mathrm{T}$ cells that express natural killer cell markers and produce immunoregulatory cytokines. ${ }^{69}$

Filarias produce the anti-inflammatory molecule ES62, which suppresses B-cell activation and proliferation by interaction with the signal transduction cascade of the B-cell antigen receptor and inhibits production of proinflammatory cytokines by interaction with the TLR signal transduction cascade. ${ }^{70}$ Further, oligosaccharides with immunomodulatory capacities such as lacto-N-neotetraose, which helminthes express on their surface, induce a subgroup of natural $\mathrm{Gr}^{+} \mathrm{CD} 11 \mathrm{~b}^{+} \mathrm{F} 4 / 80^{+}$suppressor cells, immature myeloid cells that produce IL- 10 and TGF- $\beta$ and inhibit proliferation of naive $\mathrm{CD}^{+} \mathrm{T}$ cells via IFN- $\gamma-$ dependent cell-cell contact. ${ }^{71}$ Development of derivatives of these natural immunomodulatory molecules might be of use for primary prevention against allergen-mediated diseases.

\section{Conclusion}

Enormous progress in clarifying the genetic and molecular mechanisms of allergic sensitization allows the development of novel immunomodulatory strategies aimed at primary prevention of allergen-mediated diseases. These are based either on the inhibition of their most relevant pathogenetic elements or in the induction of natural immunoregulatory mechanisms. The achievement of balance in adaptive immune responses against allergens represents the common goal of novel preventive concepts. Ultimately, these specific and curative treatment procedures shall remove symptomatic and often unspecific therapies with potentially severe side effects. The first promising experimental data are giving hope but need to be carefully validated in clinical trials for practicability, safety, and efficiency.

\section{References}

1. Isolauri EA, Huurr S, Salminen O, Impivaara O. The allergy epidemic extends beyond the past few decades. Clin Exp Allergy 2004;34:1007-10. 
2. Coffman RL. Origins of the TH1-TH2 model: a personal perspective. Nat Immunol 2006;7:539-41.

3. Kabesch M, Schedel M, Carr D, et al. IL-4/IL-13 pathway genetics strongly influence serum IgE levels and childhood asthma. J Allergy Clin Immunol 2006;117:269-74.

4. Holt PG, Thomas WR. Sensitization to airborne environmental allergens: unresolved issues. Nat Immunol 2006;6:957-60.

5. Marks GB, Mihrshahi S, Kemp AS, et al. Leader for the Childhood Asthma Prevention Study team. Prevention of asthma during the first 5 years of life: a randomized controlled trial. J Allergy Clin Immunol 2006;118:53-61.

6. Spahn JD, Szefler SJ. Childhood asthma: new insights into management. J Allergy Clin Immunol 2002;109:3-13.

7. Kurukulaaratchy RJ, Matthews S, Arshad SH. Defining childhood atopic phenotypes to investigate the association of atopic sensitization with allergic disease. Allergy 2005;60:12806.

8. Zhou B, Comeau MR, De Smedt T, et al. Thymic stromal lymphopoietin as a key initiator of allergic airway inflammation in mice. Nat Immunol 2005;6:1047-53.

9. Ito T, Wang YH, Duramad O, et al. TSLP-activated dendritic cells induce an inflammatory $\mathrm{T}$ helper type 2 cell response through OX40 ligand. J Exp Med 2005;202:1213-23.

10. Hoshino A, Tsuji T, Matsuzaki J, et al. STAT6-mediated signaling in Th2-dependent allergic asthma: critical role for the development of eosinophilia, airway hyper-responsiveness and mucus hypersecretion, distinct from its role in Th2 differentiation. Int Immunol 2004;16:1497-505.

11. Weidinger S, Klopp N, Wagenpfeil S, et al. Association of a STAT 6 haplotype with elevated serum IgE levels in a population based cohort of white adults. J Med Genet 2004;41:658-63.

12. Usui T, Preiss JC, Kanno Y, et al. T-bet regulates Th1 responses through essential effects on GATA-3 function rather than on IFNG gene acetylation and transcription. J Exp Med 2006;203:755-66.

13. Mosmann TR, Cherwinski H, Bond MW, et al. Two types of murine helper T cell clone. I. Definition according to profiles of lymphokine activities and secreted proteins. J Immunol 1986;136: 2348-57.

14. Harrington LE, Hatton RD, Mangan PR, et al. Interleukin 17producing CD4+ effector $\mathrm{T}$ cells develop via a lineage distinct from the T helper type 1 and 2 lineages. Nat Immunol 2005;6: 1023-32.

15. Park H, Li Z, Yang XO, et al. A distinct lineage of CD4 T cells regulates tissue inflammation by producing interleukin 17 . Nat Immunol 2005;6:1133-41.

16. O'Byrne PM. Cytokines or their antagonists for the treatment of asthma. Chest 2006;130:244-50.

17. Quarcoo D, Weixler S, Joachim RA, et al. Resiquimod, a new immune response modifier from the family of imidazoquinolinamines, inhibits allergen-induced Th2 responses, airway inflammation and airway hyper-reactivity in mice. Clin Exp Allergy 2004;34: 1314-20.

18. Bian T, Yin KS, Jin SX, et al. Treatment of allergic airway inflammation and hyperresponsiveness by imiquimod modulating transcription factors T-bet and GATA-3. Chin Med J 2006;119: 640-8.

19. Wang LH, Yang XY, Kirken RA, et al. Targeted disruption of stat6 DNA binding activity by an oligonucleotide decoy blocks IL-4driven $\mathrm{T}(\mathrm{H}) 2$ cell response. Blood 2000;95:1249-57.
20. Yokozeki $\mathrm{H}, \mathrm{Wu} \mathrm{MH}$, Sumi $\mathrm{K}$, et al. In vivo transfection of a cis element 'decoy' against signal transducers and activators of transcription 6 (STAT6)-binding site ameliorates IgE-mediated late-phase reaction in an atopic dermatitis mouse model. Gene Ther 2004;11:1753-62.

21. Quarcoo D, Weixler S, Groneberg D, et al. Inhibition of signal transducer and activator of transcription 1 attenuates allergeninduced airway inflammation and hyperreactivity. J Allergy Clin Immunol 2004;114:288-95.

22. Popescu F. Antisense- and RNA interference-based therapeutic strategies in allergy. J Cell Mol Med 2005;9:840-53.

23. Finotto S, De Sanctis GT, Lehr HA, et al. Treatment of allergic airway inflammation and hyperresponsiveness by antisenseinduced local blockade of GATA-3 expression. J Exp Med 2001; 193:1247-60.

24. Danahay $\mathrm{H}$, Hill S, Natt F, Owen CE. The in vitro and in vivo pharmacology of antisense oligonucleotides targeted to murine Stat6. Inflamm Res 2000;49:692-9.

25. Peng Q, Matsuda T, Hirst SJ. Signaling pathways regulating interleukin-13-stimulated chemokine release from airway smooth muscle. Am J Respir Crit Care Med 2004;169:596-603.

26. Bagasra O, Prilliman KR. RNA interference: the molecular immune system. J Mol Histol 2004;35:545-53.

27. Trian T, Girodet PO, Ousova O, et al. RNA interference decreases PAR-2 expression and function in human airway smooth muscle cells. Am J Respir Cell Mol Biol 2006;34:49-55.

28. Pazdrak K, Olszewska-Pazdrak B, Stafford S, et al. Lyn, Jak2, and Raf-1 kinases are critical for the antiapoptotic effect of interleukin 5, whereas only Raf-1 kinase is essential for eosinophil activation and degranulation. J Exp Med 1998;188:421-9.

29. Boehme SB, Sullivan SK, Crowe PD, et al. Activation of mitogenactivated protein kinase regulates eotaxin-induced eosinophil migration. J Immunol 1999;163:1611-8.

30. Duan W, Chan JHP, Hong Wong C, et al. Anti-inflammatory effects of mitogen-activated protein kinase kinase inhibitor U0126 in an asthma mouse model. J Immunol 2004;172:7053-9.

31. Hutloff A, Dittrich AM, Beier KC, et al. ICOS is an inducible T-cell co-stimulator structurally and functionally related to CD28. Nature 1999;397:263-6.

32. Chialda L, Zhang M, Brune K, Pahl A. Inhibitors of mitogenactivated protein kinases differentially regulate costimulated $\mathrm{T}$ cell cytokine production and mouse airway eosinophilia. Respir Res 2005;15:6-36.

33. Tan AH, Wong SC, Lam KP. Regulation of mouse inducible costimulator (ICOS) expression by FYN-NFATC2 and ERK signaling in T cells. J Biol Chem 2006; [In press].

34. Kaisho T, Akira S. Toll like receptor function and signaling. J Allergy Clin Immunol 2006;117:979-87.

35. Barlan I, Bahceciler NN, Akdis M, Akdis CA. Bacillus CalmetteGuerin, Mycobacterium bovis, as an immunomodulator in atopic diseases. Immunol Allergy Clin North Am 2006;26:365-77.

36. Erb KJ, Holloway JW, Sobeck A, et al. Infection of mice with Mycobacterium bovis-Bacillus Calmette-Guerin (BCG) suppresses allergen-induced airway eosinophilia. J Exp Med 1998;187:561-9.

37. Herz U, Gerhold K, Gruber C, et al. BCG infection suppresses allergic sensitization and development of increased airway reactivity in an animal model. J Allergy Clin Immunol 1998;102: $867-74$. 
38. Sayers I, Severn W, Scanga CB, et al. Suppression of allergic airway disease using mycobacterial lipoglycans. J Allergy Clin Immunol 2004;114:302-9.

39. Hunt JR, Martinelli R, Adams VC, et al. Intragastric administration of Mycobacterium vaccae inhibits severe pulmonary allergic inflammation in a mouse model. Clin Exp Allergy 2002;35: 685-90.

40. Shirtcliffe PM, Easthope SE, Weatherall M, Beasleyz R. Effect of repeated intradermal injections of heat-inactivated Mycobacterium bovis bacillus Calmette-Guérin in adult asthma. Clin Exp Allergy 2004;34:207-12.

41. Arkwright PD, David TJ. Intradermal administration of a killed Mycobacterium vaccae suspension (SRL 172) is associated with improvement in atopic dermatitis in children with moderate-tosevere disease. J Allergy Clin Immunol 2001;107:531-4.

42. Mellor AL, Baban B, Chandler PR, et al. Cutting edge: CpG oligonucleotides induce splenic CD19+ dendritic cells to acquire potent indoleamine 2,3-dioxygenase-dependent $\mathrm{T}$ cell regulatory functions via IFN Type 1 signaling. J Immunol 2005;175:5601-5.

43. Racila DM, Kline JN. Perspectives in asthma: molecular use of microbial products in asthma prevention and treatment. J Allergy Clin Immunol 2005;116:1202-5.

44. Horner AA. Toll-like receptor ligands and atopy: a coin with at least two sides. J Allergy Clin Immunol 2006;117:1133-40.

45. Schaub B, Lauener R, von Mutius E. The many faces of the hygiene hypothesis. J Allergy Clin Immunol 2006;117:969-77.

46. Peters M, Kauth M, Schwarze J, et al. Inhalation of stable dust extract prevents allergen induced airway inflammation and hyperresponsiveness. Thorax 2006;61:134-9.

47. Yang IA, Fong KM, Holgate ST, Holloway JW. The role of Toll-like receptors and related receptors of the innate immune system in asthma. Curr Opin Allergy Clin Immunol 2006;6:23-8.

48. Gerhold K, Blümchen K, Bock A, et al. Endotoxins prevent murine IgE production, Th2 immune responses and development of airway eosinophilia, but not airway hyperreactivity. J Allergy Clin Immunol 2002;110:110-6.

49. Gerhold K, Blümchen K, Franke A, et al. Exposure to endotoxin in early life and its effect on allergen sensitization in mice. J Allergy Clin Immunol 2003;112:389-96.

50. Wang Y, McCusker C. Neonatal exposure with LPS and/ or allergen prevents experimental allergic airway disease: development of tolerance using environmental antigens. J Allergy Clin Immunol 2006;118:143-51.

51. Gerhold K, Avagyan A, Seib C, et al. Prenatal initiation of endotoxin airway exposure prevents subsequent allergen-induced sensitization and airway inflammation in mice. J Allergy Clin Immunol 2006;118:666-73.

52. Bjorksten B, Sepp E, Julge K, et al. Allergy development and the intestinal microflora during the first year of life. J Allergy Clin Immunol 2001;108:516-20.

53. Moro G, Arslanoglu S, Stahl B, et al. A mixture of prebiotic oligosaccharides reduces the incidence of atopic dermatitis during the first six months of age. Arch Dis Child 2006. [In press].
54. Kalliomaki M, Salminen S, Arvilommi H, et al. Probiotics in primary prevention of atopic disease: a randomised placebocontrolled trial. Lancet 2001;357:1076-9.

55. Feleczko W, Hamelmann E. Probiotic-induced $\mathrm{T}$ regulatory mechanisms inhibit allergic sensitization and airway inflammation in a murine model of asthma. Clin Exp Allergy 2006. [In press].

56. Pohjavuori E, Viljanen M, Korpela R, et al. Lactobacillus GG effect in increasing IFN-gamma production in infants with cow's milk allergy. J Allergy Clin Immunol 2004;114:131-6.

57. Bongaerts GPA, Severijnen RSVM. Preventive and curative effects of probiotics in atopic patients. Med Hypotheses 2005;64:1089-92.

58. Li B, Samanta A, Song X, et al. Foxp3 ensembles in T-cell regulation. Immunol Rev 2007;212:99-113.

59. Akdis M, Verhagen J, Taylor A, et al. Immune responses in healthy and allergic individuals are characterized by a fine balance between allergen-specific T regulatory 1 and T helper 2 cells. J Exp Med 2004;199:1567-75.

60. Wan YY, Flavell RA. The role of cytokines in the generation and maintenance of regulatory T cells. Immunol Rev 2006;212:114-30.

61. Yamazaki S, Inaba K, Tarbell KV, Steinman RM. Dendritic cells expand antigen-specific Foxp3+CD25+CD4+ regulatory T cells including suppressors of alloreactivity. Immunol Rev 2006;212:314-29.

62. Umetsu DT, DeKruyff RH. The regulation of allergy and asthma. Immunol Rev 2006;212:238-55.

63. Stock P, Akbari O, Berry G, et al. Induction of T helper type 1-like regulatory cells that express Foxp3 and protect against airway hyper-reactivity. Nat Immunol 2004;5:1149-56.

64. Donnelly S, Dalton JP, Loukas A. Proteases in helminth- and allergen- induced inflammatory responses. Chem Immunol Allergy 2006;90:45-64.

65. Arruda LK, Santos ABR. Immunologic responses to common antigens in helminthic infections and allergic disease. Curr Opin Allergy Clin Immunol 2006;5:399-402.

66. Yazdanbakhsh $\mathrm{M}$, Wahyuni S. The role of helminth infections in protection from atopic disorders. Curr Opin Allergy Clin Immunol 2005;5:386-91.

67. Dittrich AM, Erbacher A, Diesner F, et al. Helminth infection with Litomosoides sigmodontis induces regulatory $\mathrm{T}$ cells and inhibits allergic sensitization, airway inflammation and hyperreactivity in a murine asthma model. Submitted. [2006].

68. Kitagaki K, Businga TR, Racila D, et al. Intestinal helminths protect in a murine model of asthma. J Immunol 2006;177:1628-35.

69. Mallevaey T, Zanetta JP, Faveeuw C, et al. Activation of invariant NKT cells by the helminth parasite Schistosoma mansoni. J Immunol 2006;176:2476-85.

70. Harnett W, Harnett MM. Filarial nematode secreted product ES62 is an anti-inflammatory agent: therapeutic potential of small molecule derivatives and ES62 peptide mimetics. Clin Exp Pharmacol Physiol 2006;33:511-8.

71. Terrazas LI, Walsh KL, Piskorska D, et al. The schistosome oligosaccharide lacto-n-neotetraose expands (Grl+) cells that secrete anti-inflammatory cytokines and inhibits proliferation of naïve CD4+ T-cells: a potential mechanism for immune polarization in helminth infections. J Immunol 2001;167:5294-303. 